\title{
Application of Panoramic Image Technology in Distance Teaching System
}

\author{
http://dx.doi.org/10.3991/ijet.v10i6.4848 \\ Yanqun He \\ Hunan University of Technology, Zhuzhou, China
}

\begin{abstract}
Virtual panoramic view, as a kind of realistic technology, has rapidly developed in recent years. Compared with common modeling methods, virtual panoramic view has the advantages of a low price, simplicity, and rapid development. Moreover, it has no strict requirements for data acquisition while ensuring a vivid and smooth panoramic roam. The detailed process of creating a virtual panorama is described in this paper through a design example on the campus sports teaching website, and the simple method of panoramic release to the Internet is introduced. Based on previous research, a practical virtual panoramic teaching system was developed and applied in university sports teaching. Results show that panoramic technology is feasible to some degree in sports teaching.
\end{abstract}

Index Terms-panoramic technology, distance education, physical education

\section{INTRODUCTION}

Distance teaching has been used in an overall context with the continuous maturity of digital technology and the rapid development of the Internet. In addition to traditional courseware-targeted distance education, virtual panoramic view has been gradually applied in teaching in recent years [1]. Virtual panoramic view means shooting specific scenery from different angles, recording its appearance in different periods, and then conducting technical processing to a series of photos, generally with $360^{\circ}$ reassembly technology. The pictures or videos are then released to be created on a corresponding platform based on different demands. Users can preview and study the panoramic image and video by visiting the platform to achieve different purposes [2]. The emergence and development of panoramic image roaming technology has comprehensively blended panoramic and online release technologies, and this situation solves the problem of how products can be publicized through a network. Combining digital photographing, image processing, and panoramic playing technologies results in "panoramic photographingbased virtual reality technology." As an emerging concept generated in the information age, it is currently a hot research topic in computer multimedia teaching.

Panoramic technology combines the reality of photographing and the interaction of virtual technology. In recent years, this technology has increasingly attracted attention because of its strong sense of reality and rapid generation of panoramic view, with an application scope involved in commodity advertising and promotion (virtual shopping mall or e-commerce), tourism, multimedia demonstration, real estate, and ancient architecture art, among others. Omerčević and Leonardis matched a query image to reference panoramas that depicted the same scenes that were collected and annotated with information beforehand [3]. Apivatthakakul et al. presented panoramic images produced by stitching fluoroscopic images together using an alignment grid that demonstrated the same accuracy as evaluating the coronal plane alignment of femur fractures as conventional X-rays [4]. Additionally, Lim et al. developed a cylindrical panoramic view that allows the user to obtain a clear $360^{\circ}$ vision around historical monuments in a mobile virtual heritage exploration application at a lower computational cost but with similar production quality [5].

With the continuous development of tourism ecommerce, research on the supporting or assisting environment is important. Numerous large online operators, such as Tencent, Jietu, and Quanjingke, have also successfully entered the field of panoramic virtual systems. The advantage of Tencent is that it established the SOSO streetscape by simulating Google streetscapes to achieve a virtual reality platform of urban roads. Ding et al. [6] discussed image assembly technology using multiple panoramic viewpoints. $\mathrm{Wu}$ and $\mathrm{Yu}$ [7] applied spherical panorama in campus roaming technology and solved two major problems: the generation of panorama and the design and editing of roaming space based on a KRPano engine. $\mathrm{Hu}$ et al. developed an online virtual museum experience, adopted $360^{\circ}$ panoramic technology in a paleontology museum, and shared the design concept and the process of creating scene roaming of panoramic technology [8].

Panoramic virtual roaming technology was applied to campus physical education in this study. The demands of the students' online roaming and overall framework were analyzed, and the focus was the design and realization of panoramic virtual roaming technology. The innovation in this study is the preliminary combination of panoramic technology and physical education, and the inconvenience of distance sports education and inadequate teaching conditions are avoided.

\section{ATtEMPT AT PANORAMIC TECHNOLOGY IN PHYSICAL EDUCATION}

\section{A. Design of the distance teaching system}

The design of the distance online education platform is mainly aimed at panoramic image learning technologybased reconstruction of the existing platform. In the design of the system platform, the main modules were integrated and constructed based on the existing standard, and then the panoramic image learning module was embedded into the system program to achieve the panoramic teaching function of online distance education. 
PAPER

Application of Panoramic Image Technology in Distance Teaching System

1) Design of the teaching system courseware learning module

Courseware learning mainly refers to learning text courseware, which is presented in standard text format. Text courseware is commonly released to a website by teaching management personnel. Then the content of the courseware is directly accessed through a link in the distance education platform.

Aimed at the core subsystem of physical education, a framed design model was adopted in the design. The API (Application Programming Interface) adapter was realized in the top hidden frame for communication with the LMS (Learning Management System) server. The courseware navigation provided a suggested learning sequence, but the system also allowed students to select learning contents independently based on their actual demands. The learning content and information was recorded in the background database for convenience of taking finals.

2) Design of the teaching system online video learning module

A "video" button was designed on the interface of all data, and the video data in API was used for explanation. The students could learn each chapter selectively based on their own demand. The video learning module was mainly used to assist the courseware learning of students.

\section{B. Obtaining and distributing panoramic data in the distance teaching system}

\section{1) Selection of pictures}

Generally, two methods are used to acquire the panoramic image [9]. The first method is to use a professional photographing tool to capture the scenery. The advantages of this method are its simple operation and rapid scenerytaking speed. Moreover, obtaining the finished products is easy. However, relevant hardware equipment is costly. Thus, this approach is not widely used because of its limited application. The second method is to use a household digital camera to capture scenery and then form the final finished products through assembly. However, this method has a high shooting technology requirement and requires the use of a tripod and holder. Although the second method is more complicated than the first, it is widely applied, has a low cost, and is currently the mainstream method. Hence, this study used the second method.

The selected method involved combining the camera and holder. The equipment included a Nikon digital single-lens reflex camera, a Sigma $8 \mathrm{~mm}$ fisheye lens, and a Manfrotto 303SPH holder. The shooting processes complied with the following parameters:

- Sceneries were captured in all physical education locations.

- The camera was debugged, and the appropriate aperture and shutter speed values were selected.

- After multiple experiments, the elevation angle of the camera was set at $5^{\circ}$, the camera was rotated to form a circle, and a picture was taken every $60^{\circ}$. After shooting, the holder was removed, and the ground was shot; a total of seven photos were obtained. During shooting, attention was paid to equipment stability.

2) Making the panorama

The Stitcher panoramic stitching software of French REALVIZ Company was used in this study. This software is equipped with a corresponding cracking program and supports the preservation and release of the panorama.

3) Splicing panoramic pictures

A panorama can be constituted only by splicing together the collected fisheye photos. Therefore, image splicing is one of the most important steps in panoramic generation technology. The later imaging process mainly includes three stages [10]: preprocessing of the early initial image, characteristic matching of the image, and image combination. The actual image processing stage includes preprocessing and extraction of image characteristics. The characteristic matching stage mainly aims to eliminate error matching points and match the extracted characteristic point [10]. Image combination aims to conduct secondary smoothing of the overlapped parts of the processed image and other defects to enhance the image browsing and reproduction effect. The main steps are as follows:

Step 1: The REALVIZ Stitcher 4.0 software is run.

Step 2: The Load Image command is selected in the File menu. All pictures in the panorama are loaded into the source figure window; these pictures are arranged automatically according to sequence.

Step 3: The first picture is dragged to the synthesized editing window. The second picture is also dragged. The same part of the two pictures is aligned. Then, Enter is clicked to stitch the images.

Step 4: The third picture is stitched with the second one based on Step 3.

Step 5: The Adjust All Images command is selected in the Stitch menu to adjust the torsion of the three pictures and stitch the remaining pictures.

Step 6: The remaining pictures are stitched based on Step 3.

Step 7: The final picture is selected. Close Panorama is clicked, and then the final picture is dragged to the first one for stitching.

In the panoramic display technology in this study, the column-shaped panoramic roaming technology was selected for the physical education practice. Employing the column-shaped panoramic presentation in university physical education had the advantage of different angles. Thus, students could observe the teachers' demonstration from any view and could also zoom the movement in an appropriate direction. The hot spot [1] interaction function was used to achieve rapid switching in each learning location.

\section{4) Webpage release of panoramic pictures}

The developed panoramic images were released on the Internet, and this was achieved by using mature software to make the image. The panoramic image was also realized in this example by compiling the PTViewer Java applet. PTViewer technology was realized by embedding the Java applet into the HTML page. The advantage of this technology was that it could be adapted to all operations with only appropriate changes, such as a change in distance and angle shifting. This condition is also the socalled hot-spot operation. The PTViewer Java applet was partially adjusted in this study (the parameter labels that were required for the adjusted program were fewer than before), and then the code was run. The users were required to state only the size of the browsing window and the name of the panoramic pictures browsed in the applet. PTViewer also provided the functions of Shot-Spot and Hot-Spot. Using the static hot spot provided the users with 
PAPER

an operation button to view the panorama. Meanwhile, the dynamic hot spot achieved the hot spot interaction function and shifted from a panoramic scene to the next one. In the program, $\mathrm{x}$ and $\mathrm{y}$ were the absolute coordinate val- ues of the entire picture assigned by the hot spot; the program in this case was the scene model of the final panorama. (Figures 1, 2, and 3)

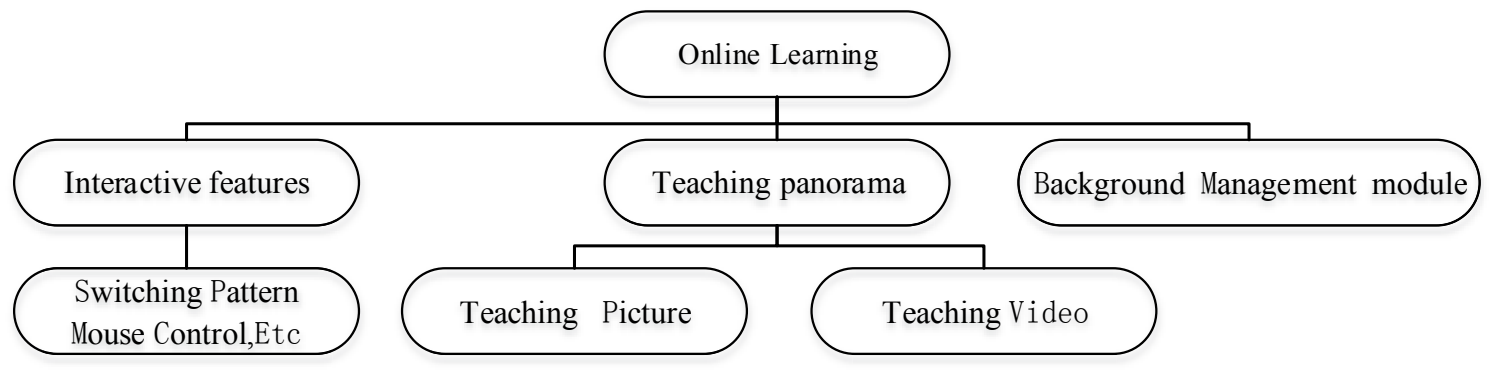

Figure 1. Functional structure diagram of panoramic teaching

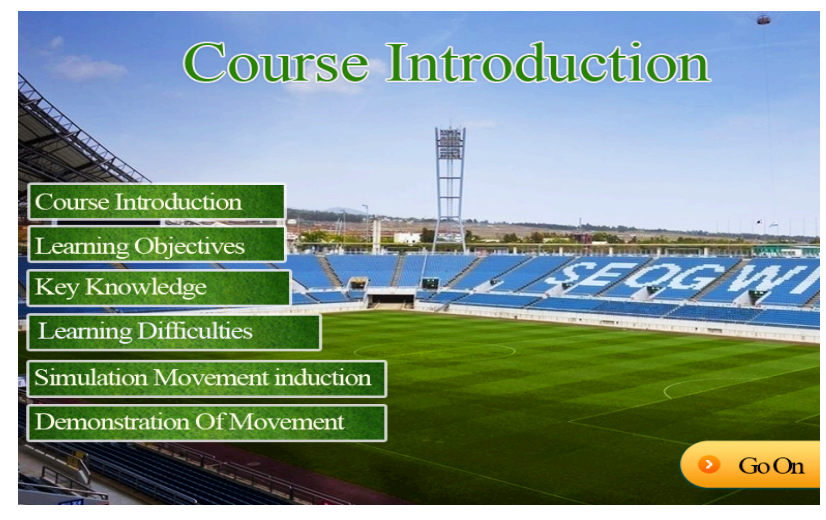

Figure 2. Main interface of distance education platform of panoramic teaching

\section{SPORTS TEACHING EXPERIMENT UNDER THE PANORAMIC ONLINE MODEL}

\section{A. Experimental objects}

A total of 104 students from two Grade 2 classes at the Hunan University of Technology were randomly selected as the research target. A total of 51 students (32 males, 19 females) from Class 1 were the experiment group, and 53 (33 males, 20 females) from Class 2 were the control group. From September 2014 to January 2015 the students in the experiment group used the panoramic online remote teaching platform for sports learning, whereas those in the control group used the ordinary teaching model for learning. The students of the two groups possessed the same knowledge content, arrangement of term and class hours, and teachers. The study was conducted in one academic term, with 56 class hours in total.

\section{B. Evaluation method of learning result}

The influence of the remote online learning system platform of the panoramic reproduction model on the sports teaching effect of Taiji boxing was comprehensively evaluated in the experiment from two aspects: (1) Evaluation of teaching satisfaction. A questionnaire method was used in the medium-term course in which students were required to fill in the teaching satisfaction questionnaire. The questionnaire content included teaching method, teaching content, class order, class atmosphere, and course interest. A five-point scoring method was used in which a higher score represented higher satisfaction. (2) Teaching effect evaluation. After completion of the course, the

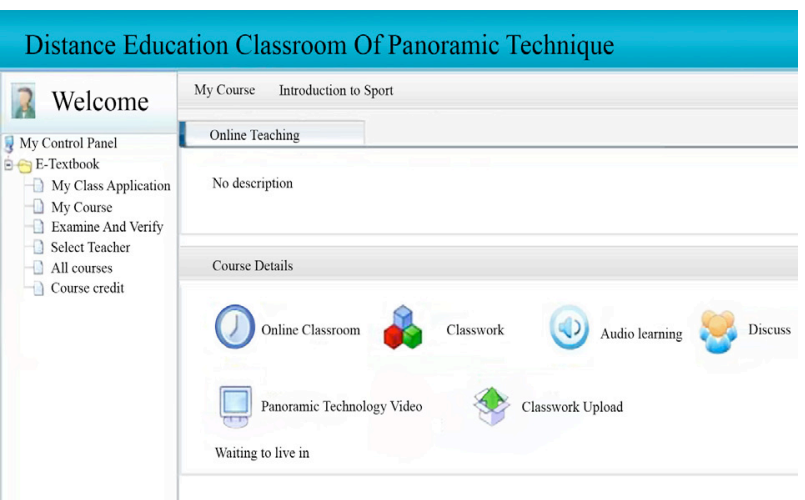

Figure 3. Interface of distance education platform of panoramic teaching

teaching evaluation team (four persons in total, excluding the teachers) scored the students' mastery of Taiji boxing. The scoring included correctness, smoothness, integrity, coordination, and performance, with a total score of 20 in each dimension. The average score of the four-person evaluation group was the final score.

\section{Statistical method}

Data were analyzed by using SPSS 15.0 statistical software, and quantitative data were expressed with mean \pm standard deviation. A comparison of the data between the two groups was examined with a t test, and the qualitative data were examined by using an $\chi 2$ test. The difference was statistically significant when $\mathrm{P}<0.05$.

\section{RESUlts}

\section{A. Comparison of general data}

The two groups were not statistically significant in terms of gender, age, height, weight, and BMI $(\mathrm{P}>0.05)$ and were comparable.

\section{B. Comparison of teaching satisfaction}

Table II shows that the score of the experiment group was higher than that of the control group in terms of teaching method, teaching content, class order, class atmosphere, and course interest, with a statistically significant difference $(\mathrm{P}<0.05)$. This result indicates that the teaching satisfaction of the experiment group was superior to that of the control group. 
PAPER

Application of Panoramic Image Technology in Distance Teaching System

TABLE I. COMPARISON OF GENERAL DATA

\begin{tabular}{|c|c|c|c|c|}
\hline Variable & Experimental Group $(n=51)$ & Control Group $(n=53)$ & $t / \chi^{2}$ & $\mathbf{P}$ \\
\hline Gender (male/female) & $32 / 19$ & $33 / 20$ & 0.003 & 0.960 \\
\hline Age (year & $18.4 \pm 1.4$ & $18.3 \pm 1.5$ & 0.351 & 0.726 \\
\hline Height (cm) & $174.8 \pm 14.6$ & $175.1 \pm 10.4$ & 0.121 & 0.904 \\
\hline Weight (kg) & $67.5 \pm 9.0$ & $68.1 \pm 9.3$ & 0.334 & 0.739 \\
\hline BMI (kg/m2) & $22.1 \pm 1.5$ & $22.2 \pm 2.3$ & 0.262 & 0.794 \\
\hline
\end{tabular}

TABLE II. COMPARISON OF TEACHING SATISFACTION

\begin{tabular}{|c|c|c|c|c|}
\hline Variable & Experimental Group $(n=51)$ & Control Group $(n=53)$ & $t$ & $\boldsymbol{P}$ \\
\hline Teaching method & $4.21 \pm 0.68$ & $3.41 \pm 0.61$ & 6.321 & 0.000 \\
\hline Teaching contents & $3.81 \pm 0.68$ & $3.49 \pm 0.58$ & 2.585 & 0.011 \\
\hline Class order & $4.01 \pm 0.71$ & $3.59 \pm 0.62$ & 3.217 & 0.002 \\
\hline Class atmosphere & $4.15 \pm 0.72$ & $3.61 \pm 0.63$ & 4.075 & 0.000 \\
\hline Course interest & $4.12 \pm 0.61$ & $3.71 \pm 0.65$ & 3.314 & 0.001 \\
\hline
\end{tabular}

TABLE III. EVALUATION OF TEACHING EFFECT

\begin{tabular}{cccc}
\hline Variable & Experimental Group (n=51) & Control Group (n=53) & \multicolumn{1}{c}{$\boldsymbol{P}$} \\
\hline Correctness & $17.8 \pm 3.1$ & $15.2 \pm 3.3$ & 4.138 \\
Smoothness & $18.7 \pm 4.5$ & $16.1 \pm 3.5$ & 3.296 \\
Integrity & $17.4 \pm 5.5$ & $15.2 \pm 3.7$ & 0.001 \\
Coordination & $18.7 \pm 4.3$ & $15.4 \pm 3.6$ & 0.018 \\
Performance & $17.2 \pm 4.1$ & $14.7 \pm 3.1$ & 3.402 \\
Total score & $89.8 \pm 13.5$ & $76.6 \pm 14.1$ & 0.000 \\
\hline
\end{tabular}

\section{Comparison of teaching effect}

Table III shows that the experiment group demonstrated five dimensions: correctness, smoothness, integrity, coordination, and performance. The total score of this group was higher than that of the control group, with a statistically insignificant difference $(\mathrm{P}<0.05)$. This result indicates that the teaching effect of the experiment group was superior to that of the control group.

Table II shows that the students who used the remote learning platform of the panoramic online model showed higher scores than the students who used the ordinary teaching model. This result is mainly due to the fact that the online teaching platform of the panoramic model provides students with relative freedom in time planning. They were provided sufficient learning time without regional limitations, and they could read and learn the sense of space of the sports field, image thought knowledge, and queue arrangement, among others. All these conditions enabled the students to learn the required knowledge easily. Thus, their scores were higher than those of students who took ordinary courses. The panorama stimulated the students' creative thinking. The panorama encouraged the students to perform actively in class, ensuring a good atmosphere, class order, and concentrated attention. Moreover, the panorama cultivated the ability of students to learn and provided opportunities for them to exercise their skills. Accordingly, the students' class satisfaction and teaching effect, which were evaluated based on the correctness, smoothness, integrity, coordination, and performance of Taiji boxing teaching, were improved.

\section{CONCLUSIONS}

As an important development direction, the combination of panoramic virtual roaming with online roaming will be more frequently used in the teaching system. A simple panoramic online virtual sports teaching roaming system was realized in this study. The function of this system basically met the demand of a panoramic online learning system. However, with the continuous expansion of scale, increasing number of students, and increasing number of teaching sites in the future, an increase of models will be a challenge to the development of the technology. With people's increasing demands for panoramic virtual roaming and the continuous development of panoramic online virtual roaming technology, a balance between technology and demand should be achieved.

\section{REFERENCES}

[1] Sim D.Y., Kim Y.M., "Detection and compression of moving objects based on new panoramic image modeling," Image and $\mathrm{Vi}$ sion Computing, vol. 27, no. 10, pp. 1527-1539, September 2009. http://dx.doi.org/10.1016/j.imavis.2009.02.009

[2] Brown M., Lowe D.G., "Automatic panoramic image stitching using invariant features," International Journal of Computer Vision, vol. 74, no. 1, pp. 59-73, August 2007. http://dx.doi.org/10.1007/s11263-006-0002-3

[3] Omerčević D., Leonardis A., "Hyperlinking reality via camera phones," Machine Vision and Applications. vol. 22, no. 3, pp. 521534, May 2011.

[4] Apivatthakakul T., Duanghakrung M., Luevitoonvechkit S., Patumasutra S., "Intraoperative panoramic image using alignment grid, is it accurate?" Arch Orthop Trauma Surg, vol. 133, no. 7, pp 953-959, May 2013. http://dx.doi.org/10.1007/s00402-013-1765-y

[5] Lim C.K., Tan K.L., Talib A.Z., “A Low-Cost Method for Generating Panoramic Views for a Mobile Virtual Heritage Application," Communications in Computer and Information Science, vol. 194, pp. 501-512, 2011. http://dx.doi.org/10.1007/978-3-64222603-8 44

[6] Ding F., Wan Y., Lei Y., Wang L., Zhang B.F., Shi T.Y., et al., "3D Panorama-based Online Roaming and GIS Integration Research and Development," Journal of Nankai University (natural science edition), vol. 47, no. 4, pp. 54-58, August 2014. 
[7] Wu G., Yu W., "Exploration and Realization of Virtual Campus 3D Panoramic Roaming System," Modern Education Technology, vol. 23, no. 5, pp. 122-126, May 2013.

[8] Hu J., Yang J.J, Meng J.X., "Design and Realization of Museum Network Virtual Display based on Panoramic Technology," China Information Technology Education, vol. 24, no. 1, pp. 83-86, January 2015.

[9] Zhang S.B., Rao D., "Online Real-time Debugging Model of Panoramic Data based on Named Variables," Computer Engineering and Science, vol. 34, no. 1, pp. 178-182, January 2012.

[10] Liu Q., Liu J.G., Yan J., " $360^{\circ}$ Streetscape Map Modeling and Online Release Technology Analysis," Geographical Space Information, vol. 13, no. 1, pp. 48-49+58, February 2015.

\section{AUTHOR}

Yanqun He (Corresponding author) is a lecturer at the Physical Education Institute, Hunan University of Technology, Zhuzhou, 412007, China. Her research interests include distance teaching and physical education (yanqunhe@yeah.net)

Submitted 07 July 2015. Published as resubmitted by the author 15 November 2015. 